\title{
Vagolytic atropine attenuates cerebral vasodilation response during acute orthostatic hypotension
}

\author{
Woo-Jong Choi ${ }^{1}$, Kichang Lee ${ }^{2}$, Young-Kug Kim ${ }^{1}$, Kyo-Joon Song ${ }^{3}$, \\ Sung-Moon Jeong ${ }^{1}$, and Gyu-Sam Hwang ${ }^{1}$ \\ ${ }^{I}$ Department of Anesthesiology and Pain Medicine, Laboratory for Cardiovascular Dynamics, Asan Medical Center, \\ University of Ulsan College of Medicine, Seoul, Korea, ${ }^{2}$ Harvard-MIT Division of Health Sciences and Technology, \\ Massachusetts Institute of Technology, MA, USA, ${ }^{3}$ Department of Anesthesiology and Pain Medicine, VHS Medical \\ Center, Seoul, Korea
}

Background: Atropine is an anticholinergic drug which is commonly used in clinical practice. The effect of parasympathetic block with atropine on dynamic cerebrovascular regulation remains unclear. This study was aimed to identify effects of vagolytic atropine on cerebrovascular response during acute orthostatic hypotension in humans.

Methods: Continuous middle cerebral blood flow velocity (CBFV, transcranial Doppler) and arterial blood pressure (ABP, Finometer) were measured during a sit-to-stand procedure in 10 healthy subjects with placebo and vagolytic $(10 \mu \mathrm{g} / \mathrm{kg})$ doses of atropine. Cerebral vascular tone was assessed by cerebrovascular resistance (CVR $=\mathrm{ABP} / \mathrm{CBFV})$. Dynamic cerebral autoregulation was also assessed by transfer function analysis of ABP and CBFV.

Results: During the standing session, ABP fell to a similar extent in both groups by an average of 23 to $25 \mathrm{mmHg}$ (26\% to $29 \%)$. CBFV also fell in all subjects but significantly more in vagolytic atropine $(-15.0 \pm 7.0 \mathrm{~cm} / \mathrm{s})$ compared with placebo $(-12.0 \pm 5.8 \mathrm{~cm} / \mathrm{s}, \mathrm{P}<0.05)$. CVR was decreased significantly in the placebo group during posture change $(1.56 \pm$ 0.44 vs. $1.38 \pm 0.38, \mathrm{P}<0.05)$, in contrast, lesser decreased in the atropine group $(1.60 \pm 0.50$ vs. $1.53 \pm 0.42, \mathrm{P}=0.193)$. Transfer function coherence in the very-low-frequency range was significantly increased in the atropine group during the standing session $(0.55 \pm 0.14)$, compared with the sitting session $(0.45 \pm 0.14, \mathrm{P}=0.006)$.

Conclusions: These data present that vagolytic atropine attenuates cerebral vasodilation response to acute orthostatic hypotension, suggesting the use of atropine may need care in patients with cerebrovascular disease with vagal impairment.

Key Words: Atropine, Autoregulation, Cerebral blood flow, Orthostatic hypotension.

Corresponding author: Gyu-Sam Hwang, M.D., Ph.D.

Department of Anesthesiology and Pain Medicine, Laboratory for Cardiovascular Dynamics, Asan Medical Center, University of Ulsan College of Medicine, 88, Olympic-ro 43-gil, Songpa-gu, Seoul 05505, Korea Tel: 82-2-3010-3868, Fax: 82-2-3010-6790

E-mail: kshwang@amc.seoul.kr

Received: March 6, 2015.

Revised: April 29, 2015.

Accepted: June 52015.

Korean J Anesthesiol 2015 December 68(6): 594-602 http://dx.doi.org/10.4097/kjae.2015.68.6.594

\section{Introduction}

The healthy human maintains a relatively constant cerebral blood flow $(\mathrm{CBF})$ in response to changes in arterial blood pressure $(\mathrm{ABP})$; the process by which this occurs is termed cerebral autoregulation (CA) [1]. Impaired dynamic CA, referring to $\mathrm{CBF}$ responses to rapid $\mathrm{ABP}$ changes occurring over seconds, may contribute to the pathophysiology of vasovagal or neurally mediated syncope, associated with an acute reduction in CBF.

It has long been known that the parasympathetic nervous

(c) This is an open-access article distributed under the terms of the Creative Commons Attribution Non-Commercial License (http://creativecommons.org/ licenses/by-nc/4.0/), which permits unrestricted non-commercial use, distribution, and reproduction in any medium, provided the original work is properly cited. 
system may play a role in cerebrovascular regulation [2,3]. In addition, the pathophysiology of Alzheimer's disease is characterized by a cholinergic denervation of cerebral microvessels and a diminished cerebral perfusion $[4,5]$.

In more recent human studies [6,7], cholinergic blockade by glycopyrrolate abolished the exercise-induced increase in cerebral perfusion, and impaired cerebral autoregulation at frequencies above $0.04 \mathrm{~Hz}$. Glycopyrrolate was used to block muscarinic receptors on the endothelium of cerebral vessel because it does not penetrate the blood-brain barrier in contrast to atropine [8]. However, cerebral vessels are surrounded by nerve fibers that originate from peripheral nerve ganglia and intrinsic brain neurons, respectively. The regulation of cerebrovascular tone is under control of the intrinsic innervations, as well as the extrinsic innervations [9]. Thus, atropine may be better than glycopyrrolate to evaluate parasympathetic role in cerebrovascular tone.

Atropine is an anticholinergic drug which is commonly used for treatment of bradycardia, cardiopulmonary resuscitation, and organophosphate poisoning. Specially, vagolytic $(10-15 \mu \mathrm{g} / \mathrm{kg})$ dose of atropine which is sufficient to block parasympathetic nervous system [10] is often used clinically in urgent situations. However, the effect of atropine on CA is little known in human. Therefore, we hypothesized that cholinergic block with atropine would attenuate cerebral vasodilation response during acute orthostatic hypotension. We evaluated dynamic CA and cerebrovascular resistance (CVR) during standing after administration of atropine in human.

\section{Materials and Methods}

\section{Subjects}

Ten healthy subjects ( 5 men and 5 women) with a mean age of $29 \pm 3$ years, height of $169 \pm 8 \mathrm{~cm}$, and weight of $61 \pm 12 \mathrm{~kg}$ participated in this study. All subjects were nonsmokers and normotensive. Subjects were instructed to refrain from consuming caffeinated or alcoholic beverages at least 24 hours prior to study. The experimental protocol was approved by the Institutional Review Board of our hospital, and all subjects were verbally informed of the intent and procedures of the study with written consent obtained prior to data collection. The study was registered with the Clinical Research Information Service (number KCT0000211).

\section{Instrumentation}

Subjects were instrumented for heart rate measurement on an electrocardiography lead II (Hewlett-Packard 78352A, Palo Alto, USA) and beat-by-beat photoplethysmographic ABP (Finometer, Finapres Medical Systems BV, Amsterdam, Netherland). End-tidal $\mathrm{CO}_{2}$ was measured via a nasal cannula using a mass spectrometer (Marquette Electronics, Milwaukee, WI, USA). A 2-MHz Doppler probe (Companion III, Viasys Healthcare, Madison, WI, USA) was placed over the temporal window to measure blood flow velocity in the middle cerebral artery (MCA) [11] with a probe fixation device used to stabilize the Doppler probe for the duration of the study.

All physiological signals were digitized at $500 \mathrm{~Hz}$ using a commercially available data acquisition converter (Windaq, Dataq Instruments, Akron, OH, USA) with a personal computer and stored for subsequent off-line analysis. A 25-gauge catheter was inserted into a forearm vein for drug administration.

\section{Protocol}

This study was randomized and double-blind comparison of subjects between the vagolytic $10 \mu \mathrm{g} / \mathrm{kg}$ atropine and placebo (an equal volume with normal saline) groups [10]. At least, 14 days were allowed between experiments. All subjects received two types of infusions. Ten min after injection of atropine or placebo, 6 min of baseline data were collected in the sitting position.

Orthostatic hypotension was induced to assess dynamic CA by an active sit-to-stand procedure as described previously [12]. Subjects sat in a straight-backed chair with their legs elevated at a $90^{\circ}$ angle for $5 \mathrm{~min}$, then stood upright for $1 \mathrm{~min}$. The initiation of standing was timed from the moment both feet touched the floor. After these two sit-to-stand trials, subjects performed a 6 min standing for the transfer function analysis of $\mathrm{ABP}$ and CBFV signals (Fig. 1). During the studies, the hand with the Finapres cuff was held at heart level to eliminate hydrostatic pressure effects. Respiration was paced by aural entrainment at 0.25 $\mathrm{Hz}$ (15 breaths/min) during all data collection periods to control normocapnia (the end-tidal partial pressure of $\mathrm{CO}_{2}$ between 37 and $40 \mathrm{mmHg}$ ) and to permit the spectral analysis of physiological variables, without the influence of respiratory cycles.

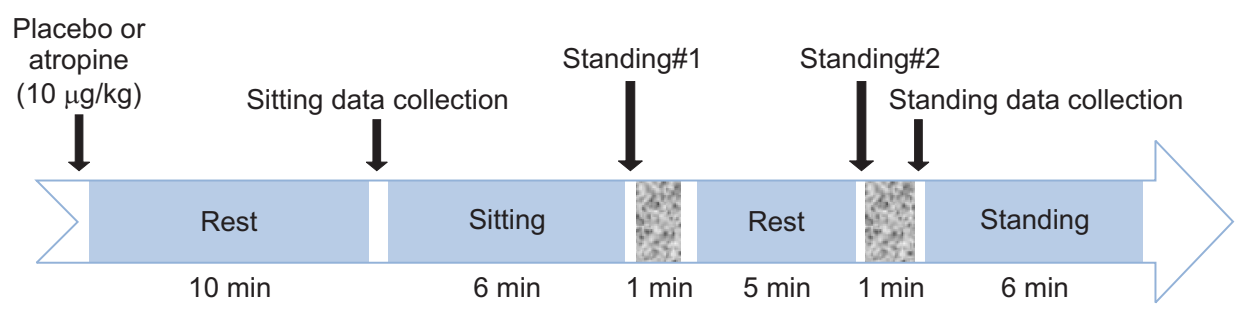

Online access in http://ekja.org
Fig. 1. General experimental protocol used for application of the sit-to-stand procedure. The study was a randomized, double-blind comparison both $10 \mu \mathrm{g} / \mathrm{kg}$ atropine and placebo (an equal volume of normal saline) groups. 


\section{Data processing and analysis}

Off-line analysis was done using a MATLAB program (The MathWorks, Natick, MA, USA). Beat-to-beat R-R intervals (RRI), mean values of ABP (MAP) and cerebral blood flow velocity $(\mathrm{CBFV})$ were determined from the $\mathrm{R}$ wave of the ECG and the maximum and minimum of ABP or CBFV (Fig. 2). To evaluate the beat-to-beat dynamics of $\mathrm{ABP}$ and $\mathrm{CBFV}$ responses to acute posture changes, the differences were calculated between the baseline value measured during the sitting position (average over a period of 50 seconds) and the value during standing at the nadir of $A B P$ (average of 5 values surrounding the nadir) for both MAP and CBFV $[12,13]$. Then these values for each group were averaged. We also computed these changes as a percentage of the baseline value.

Cerebrovascular response to transient orthostatic hypotension was assessed by calculation of the absolute and percent change in $\mathrm{CVR}$ ( $\mathrm{ABP} / \mathrm{CBFV}$ ) from the sitting position (average of 50 seconds) to the nadir of ABP during standing (average of 5 values) [12].

To evaluate the effect of atropine on the dynamic CA, coherence and transfer function analysis using the ABP and CBFV signals' autospectra [14] during the 5-min sitting and stand periods were also performed for each trial. Coherence $(\mathrm{C})$ of the frequency content (f) between the $\mathrm{ABP}$ and $\mathrm{CBFV}$ time series was calculated from the power spectra $(\mathrm{P})$ for the sitting position using the following formula:

$$
\mathrm{C}_{x y}(\mathrm{f})=\frac{\left|\mathrm{P}_{x y}(\mathrm{f})\right|^{2}}{\mathrm{P}_{x x}(\mathrm{f}) \mathrm{P}_{y y}(\mathrm{f})}
$$

where $\chi$ is the input signal (mean ABP) and $y$ is the output $(\mathrm{CBFV})$. The transfer function gain $(\mathrm{H})$ was also computed for the sitting position using the following formula:

$$
\mathrm{H}_{x y}(\mathrm{f})=\left|\frac{\mathrm{P}_{x y}(\mathrm{f})}{\mathrm{P}_{x x}(\mathrm{f})}\right|
$$

This method assesses autoregulation by examining the relative change in beat-by-beat $\mathrm{CBFV}$ with each beat-by-beat change in ABP.

The spectral power of $\mathrm{ABP}$ and $\mathrm{CBFV}$, mean value of transfer function gain, phase and coherence function were calculated in the very-low-frequency (VLF, 0.02-0.07 Hz), low-frequency (LF, $0.07-0.15 \mathrm{~Hz}$ ), and high-frequency ( $\mathrm{HF}, 0.15-0.30 \mathrm{~Hz}$ ) ranges. These ranges reflect different patterns of the dynamic pressureflow relationship $[14,15]$. CA maintains CBFV relatively constant by using changes in CVR to buffer changes in ABP that would otherwise cause large fluctuations in CBFV. In the very-lowfrequency ranges, the damping effect of $\mathrm{CA}$ on changes in $\mathrm{ABP}$ is effective [14]. Coherence function between 0 and 1 reflects the linear relation between ABP and CBFV. If low coherence $(<0.5)$ observes at very-low-frequency (autoregulatory) ranges, this indicates nonlinear property of CA. Phase reflects the temporal relation between oscillations of $\mathrm{ABP}$ (input function) and CBFV (output function). A phase decreases during conditions of compromised cerebral circulation. Transfer function gain as the ratio of the amplitudes of CBFV and $\mathrm{ABP}$ was taken to reflect the ability of the cerebrovascular bed to buffer changes in $\mathrm{CBFV}$ induced by transient changes in ABP. If autoregulation is functioning properly, changes in ABP cause minimal changes in

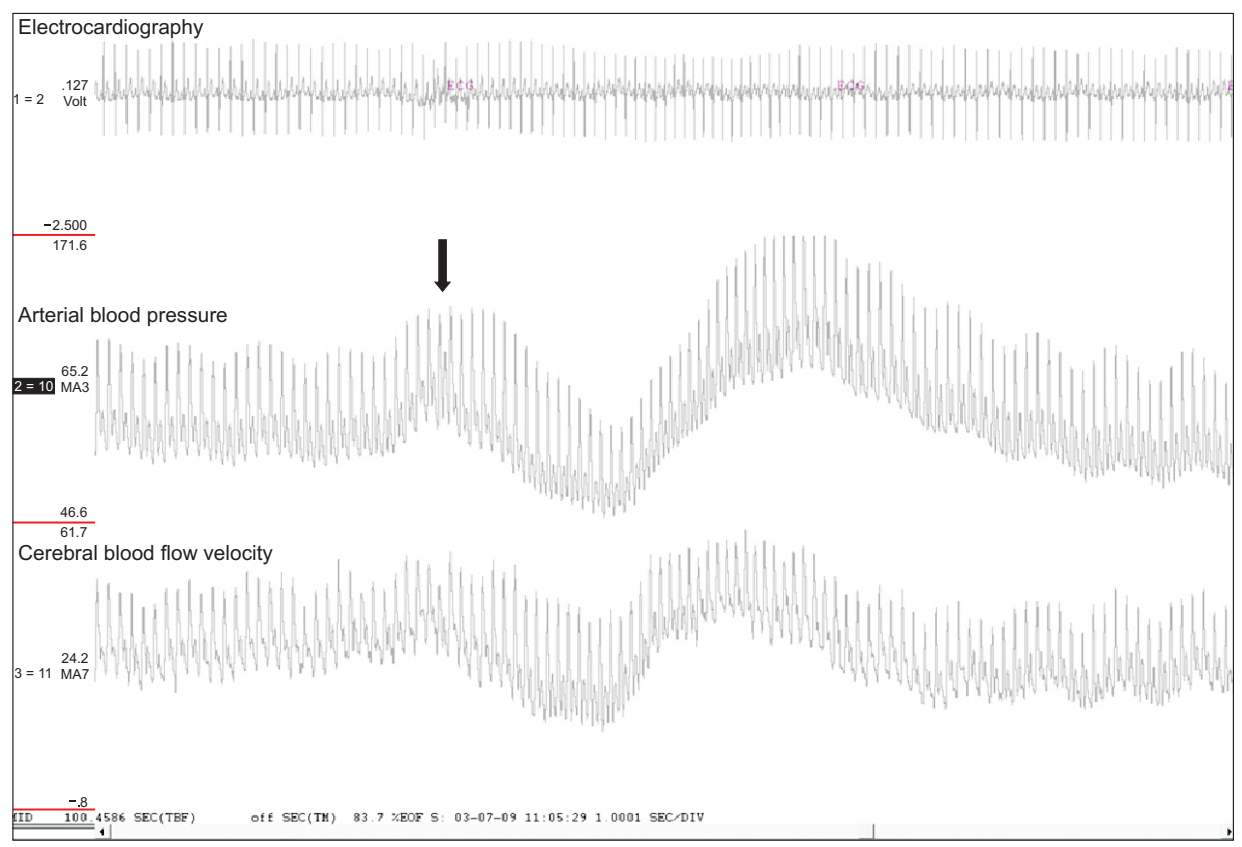

Fig. 2. Arterial blood pressure and cerebral blood flow velocity response during the sit-to-stand procedure. Continuous beat-to-beat arterial blood pressure and cerebral blood flow velocity measured during the sit-to-stand procedure after atropine injection in a healthy subject. The black arrow represents point at which stand was initiated. 
CBFV, and thus transfer function gains should be low. When autoregulation is impaired, changes in ABP cause large changes in flow and thus gains should be high. Thus, a larger gain indicates that any given changes in blood pressure leads to a larger change in cerebral blood flow, implying impaired autoregulation [16,17].

Autonomic system response to each experimental condition was assessed with a power spectral density (Welch's periodogram) of R-R interval (RRI) variability and spontaneous baroreflex function, which was evaluated by 1) frequency-domain transfer function analysis of RRI and systolic ABP in the lowfrequency $\left(\mathrm{BRS}_{\mathrm{LP}} 0.04-0.15 \mathrm{~Hz}\right.$ ) and 2) time-domain baroreflex sequences $\left(B_{\text {Req }}\right)$ analysis of RRI and systolic $A B P$, as described previously [18].

\section{Statistics}

All data are expressed as mean values \pm SD. All scaled variables were tested for normality using the Shapiro-Wilk test. Because the distribution of both LF and HF components of RRI variability were skewed, the data were analyzed after a natural logarithmic (ln) transformation. To determine the effects of position and cholinergic blockade, hemodynamic variables, transfer function coherence, gain, phase, and spectral power of RRI, $\mathrm{ABP}, \mathrm{CBFV}$ were compared using two way repeated-measures analysis of variance with Bonferroni test for multiple comparisons and Friedman's test with Tukey post hoc test, as appropriate. The differences of all other variables were compared using paired t-tests. All statistical procedures were performed using personal computer-based software (Sigmaplot 11.0, Systat Software, Inc., CA, USA). Statistical significance level was accepted at $\mathrm{P}<0.05$.

\section{Results}

\section{Cardiovascular and cerebral hemodynamics}

Average $\mathrm{BP}, \mathrm{CBFV}$, and CVR during sitting as well as the first 60 seconds of standing are shown in Fig. 3. A summary of the
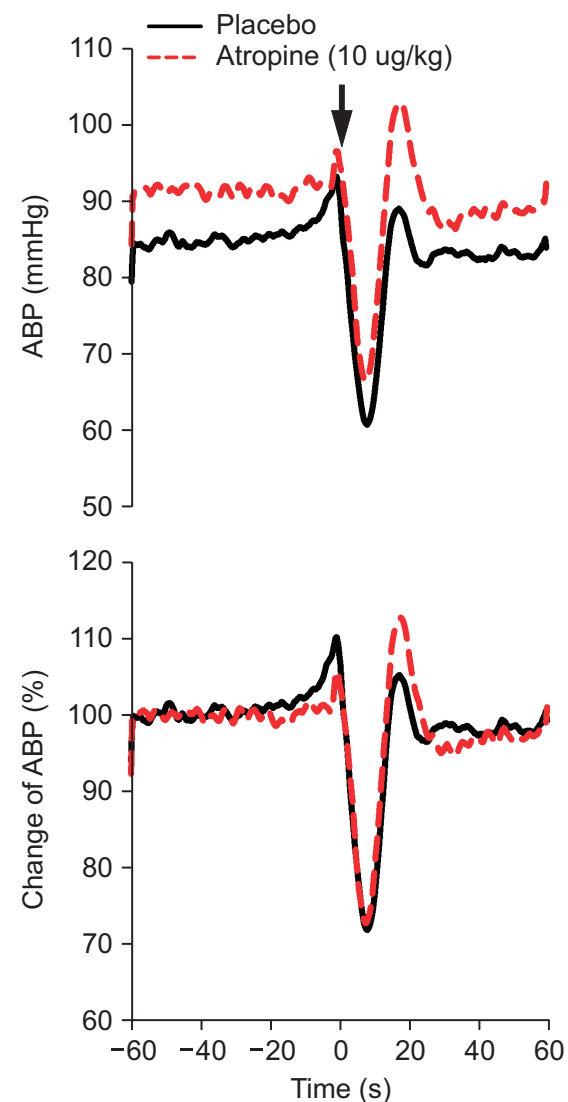
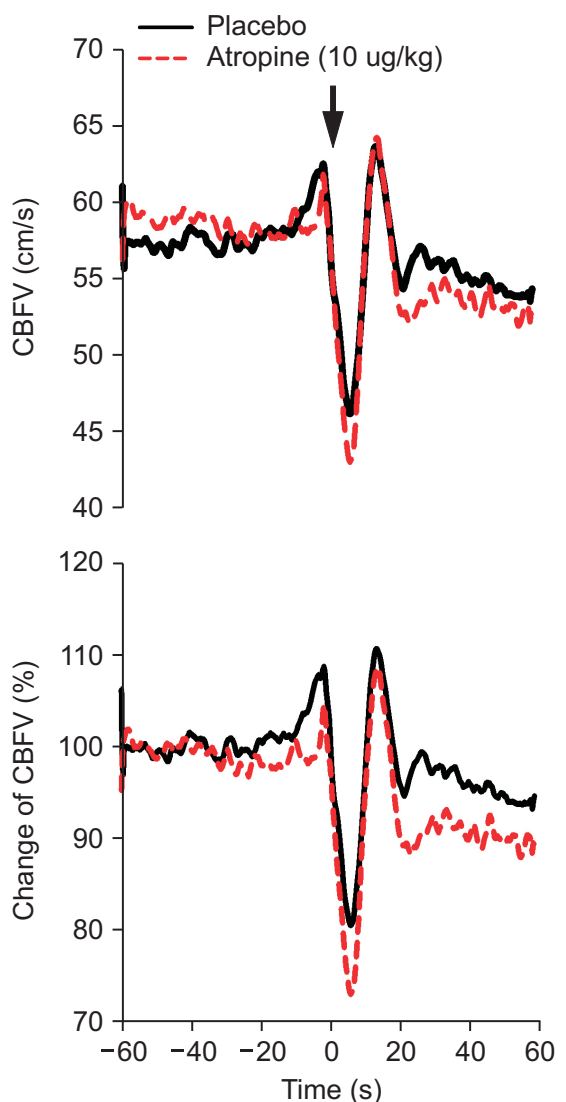
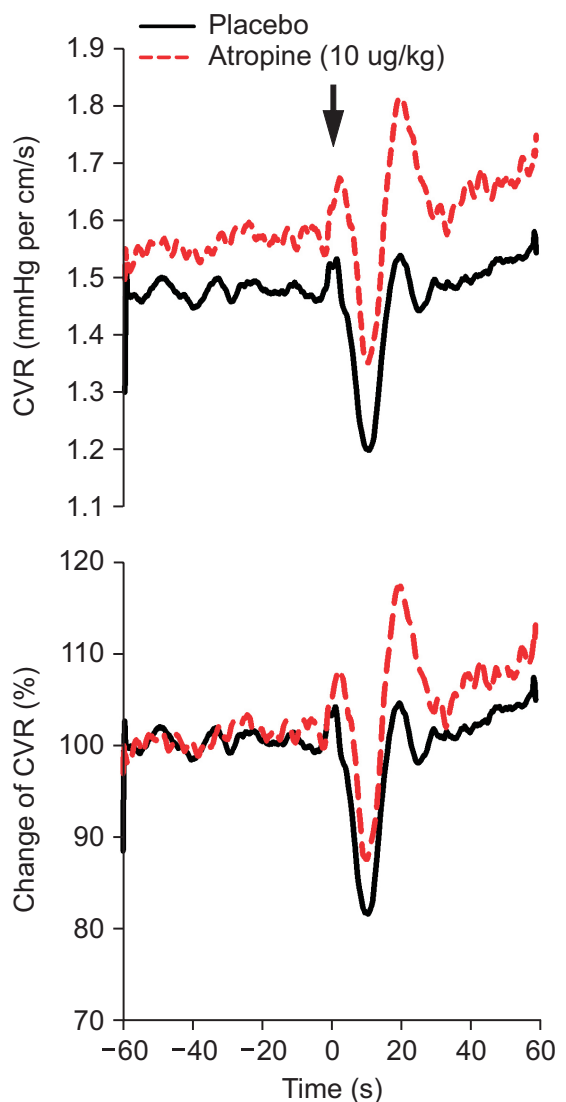

Fig. 3. Group-averaged cerebrovascular responses during the sit-to-stand procedure. Normalized arterial blood pressure (ABP, lower right), cerebral blood flow velocity (CBFV, lower middle) and cerebral vascular resistance (CVR, lower left). The black arrows and time 0 represents point at which subject's feet touched the floor and stand was initiated. Note that at the nadir of ABP after standing, the change of ABP was not different in both groups. However, $\mathrm{CBFV}$ was significantly more decreased in the atropine group than the placebo $(\mathrm{P}=0.028)$. CVR was decreased significantly in the placebo group during posture change $(\mathrm{P}<0.05)$, in contrast, lesser decreased in the atropine group. 
Table 1. Cardiovascular and Cerebral Hemodynamics to Postural Change

\begin{tabular}{|c|c|c|c|c|c|}
\hline & & Sitting & Standing & Difference & $\%$ Difference \\
\hline \multirow[t]{2}{*}{ HR (bpm) } & Placebo & $77.7 \pm 10.4$ & $99.8 \pm 11.8^{\dagger}$ & $22.1 \pm 6.7$ & $29.2 \pm 11.0$ \\
\hline & Atropine & $97.8 \pm 10.9^{*}$ & $118.8 \pm 10.1^{*, \dagger}$ & $21.0 \pm 3.0$ & $21.8 \pm 4.5$ \\
\hline \multirow[t]{2}{*}{$\mathrm{ABP}(\mathrm{mmHg})$} & Placebo & $87.3 \pm 10.7$ & $62.2 \pm 9.5^{\dagger}$ & $-25.0 \pm 8.2$ & $-28.6 \pm 8.3$ \\
\hline & Atropine & $92.4 \pm 15.6$ & $67.3 \pm 11.4^{\dagger}$ & $-25.1 \pm 11.1$ & $-26.5 \pm 9.2$ \\
\hline \multirow[t]{2}{*}{$\mathrm{CBFV}(\mathrm{cm} / \mathrm{s})$} & Placebo & $59.5 \pm 15.8$ & $47.4 \pm 12.9^{\dagger}$ & $-12.0 \pm 5.8$ & $-19.6 \pm 8.4$ \\
\hline & Atropine & $61.2 \pm 16.3$ & $46.3 \pm 12.0^{\dagger}$ & $-15.0 \pm 7.0^{*}$ & $-23.7 \pm 8.5$ \\
\hline \multirow[t]{2}{*}{$\mathrm{CVR}(\mathrm{mmHg} / \mathrm{cm} / \mathrm{s})$} & Placebo & $1.56 \pm 0.44$ & $1.38 \pm 0.38^{\dagger}$ & $-0.18 \pm 0.22$ & $-10.5 \pm 10.3$ \\
\hline & Atropine & $1.60 \pm 0.50$ & $1.53 \pm 0.42$ & $-0.08 \pm 0.14$ & $-3.3 \pm 6.7^{*}$ \\
\hline
\end{tabular}

Values are mean \pm SD. The value of standing is calculated at the nadir of ABP. HR: heart rate, ABP: arterial blood pressure, CBFV: cerebral blood flow velocity, CVR: cerebrovascular resistance. ${ }^{*} \mathrm{P}<0.05$ vs. placebo. ${ }^{\dagger} \mathrm{P}<0.05$ vs. Sitting.

Table 2. Spectral Power Analysis of R-R Intervals, Blood Pressure and Cerebral Blood Flow Velocity

\begin{tabular}{llcc}
\hline & & Sitting & Standing \\
\hline $\ln \mathrm{LF}_{\mathrm{RRI}}, \mathrm{ms}^{2}$ & Placebo & $4.36 \pm 0.67$ & $4.09 \pm 1.12$ \\
& Atropine & $2.44 \pm 0.73^{*}$ & $2.61 \pm 1.32^{*}$ \\
$\ln \mathrm{HF}_{\mathrm{RRI}}, \mathrm{ms}^{2}$ & Placebo & $3.38 \pm 0.86$ & $2.40 \pm 1.00^{\dagger}$ \\
& Atropine & $0.11 \pm 1.05^{*}$ & $-0.84 \pm 1.09^{*}$ \\
$\mathrm{LF}_{\mathrm{ABP}}, \mathrm{mmHg}^{2}$ & Placebo & $1.93 \pm 0.80$ & $2.25 \pm 0.94$ \\
& Atropine & $2.04 \pm 1.20$ & $4.50 \pm 2.89^{*}+$ \\
$\mathrm{HF}_{\mathrm{ABP}}, \mathrm{mmHg}^{2}$ & Placebo & $0.23 \pm 0.18$ & $1.00 \pm 0.82^{\dagger}$ \\
& Atropine & $0.48 \pm 0.36^{*}$ & $0.71 \pm 0.60$ \\
$\mathrm{LF}_{\mathrm{CBFV}}, \mathrm{cm}^{2} / \mathrm{s}^{2}$ & Placebo & $1.41 \pm 0.97$ & $1.60 \pm 0.57$ \\
& Atropine & $1.27 \pm 0.77$ & $3.24 \pm 2.87^{\dagger}$ \\
$\mathrm{HF}_{\mathrm{CBFV}}, \mathrm{cm}^{2} / \mathrm{s}^{2}$ & Placebo & $0.40 \pm 0.38$ & $1.23 \pm 0.84^{\dagger}$ \\
& Atropine & $0.88 \pm 1.26$ & $1.02 \pm 1.16$ \\
$\mathrm{LF} / \mathrm{HF}$ & Placebo & $3.28 \pm 2.74$ & $8.28 \pm 9.91^{\dagger}$ \\
& Atropine & $11.52 \pm 9.14^{*}$ & $40.60 \pm 41.95^{*}+$ \\
$\mathrm{BRS}_{\mathrm{LF}}$ & Placebo & $5.10 \pm 1.74$ & $4.35 \pm 1.19$ \\
$\mathrm{BRS}_{\text {seq }}$ & Atropine & $1.83 \pm 0.65^{*}$ & $1.79 \pm 1.01^{*}$ \\
& Placebo & $8.32 \pm 3.44$ & $6.15 \pm 3.53$ \\
& Atropine & $2.81 \pm 1.33^{*}$ & $2.03 \pm 1.17^{*}$ \\
\hline
\end{tabular}

Values are mean \pm SD. ABP: arterial blood pressure, CBFV: cerebral blood flow velocity, RRI: R-R interval, ln: natural logarithmic, LF: lowfrequency, HF: high-frequency, $\mathrm{BRS}_{\mathrm{LF}}$ : baroreflex sensitivity in lowfrequency, $\mathrm{BRS}_{\text {seq }}$ : baroreflex sensitivity sequences. ${ }^{*} \mathrm{P}<0.05$ vs. placebo. ${ }^{\dagger} \mathrm{P}<0.05$ vs. Sitting.

cardiovascular responses to atropine and standing is presented in Table 1. In the sitting position, compared with the placebo group, HR increased significantly in the atropine group $(\mathrm{P}<0.05)$. $\mathrm{ABP}, \mathrm{CBFV}$, and CVR were unchanged by atropine administration.

At the nadir of ABP after standing, HR increased, ABP decreased similarly in both groups. However, $\mathrm{CBFV}$ was significantly more decreased in the atropine group than the placebo $(\mathrm{P}=0.028)$. Thus, CVR was decreased significantly in the placebo group during posture change ( $1.56 \pm 0.44$ vs. $1.38 \pm 0.38, \mathrm{P}<0.05)$, in contrast, lesser decreased in the atropine group (1.60 \pm 0.50 vs. $1.53 \pm 0.42, \mathrm{P}=0.193)$. The percent changes in CVR was also significantly lesser in the atropine group $(-3.3 \pm 6.7 \%)$ than the placebo group $(-10.5 \pm 10.3 \%, \mathrm{P}=0.039)$.

$\mathrm{ABP}$ was fully recovered 1 minute after standing in both groups. But, the decreased CBFV during standing was partially recovered 1 minute after standing in the atropine group (53.4 $\pm 0.7 \mathrm{~cm} / \mathrm{sec}, 90 \%$ of the sitting position) compared with in the placebo group $(55.2 \pm 0.9 \mathrm{~cm} / \mathrm{sec}, 96 \%$ of the sitting position, $\mathrm{P}<0.01$ ) (Fig. 3).

\section{Spectral and transfer function analysis}

Spectral power densities of RRI, ABP and CBFV during sitting and standing sessions are shown in Table 2 and Fig. 4. In the sitting position, $\ln \mathrm{LF}_{\mathrm{RRI}}$ spectral power in the atropine group was significantly lower than in the placebo $(\mathrm{P}<0.05) . \mathrm{LF}_{\mathrm{ABP}}$ and $\mathrm{LF}_{\mathrm{CBFV}}$ spectral powers were not different in both groups. During posture change, ln $\mathrm{LF}_{\mathrm{RRI}}$ spectral power was not change in both groups. However, $\mathrm{LF}_{\mathrm{ABP}}$ and $\mathrm{LF}_{\mathrm{CBFV}}$ spectral powers were significantly increased in the atropine group $(\mathrm{P}<0.05$, respectively) compared with the sitting position. $\mathrm{LF}_{\mathrm{ABP}}$ spectral power in the standing position was higher significantly in the atropine group than the placebo $(\mathrm{P}=0.008)$.

In the sitting position, LF/HF of RRI variability in the atropine group was significantly higher than the placebo $(\mathrm{P}<0.05)$. During posture change, LF/HF of RRI variability was significantly increased in both groups $(\mathrm{P}<0.05$, respectively) compared with the sitting position.

In the sitting position, $\mathrm{BRS}_{\mathrm{LF}}$ and $\mathrm{BRS}_{\mathrm{seq}}$ were significantly lower in the atropine group than the placebo group $(\mathrm{P}<0.05$, respectively). During posture change, $\mathrm{BRS}_{\mathrm{LF}}$ and $\mathrm{BRS}_{\mathrm{seq}}$ were not significantly changed in both groups.

Fig. 5 shows transfer function analysis of beat-to-beat changes in $\mathrm{ABP}$ and $\mathrm{CBFV}$ during both sitting and standing sessions. Coherence in the VLF range remained below 0.5 in the placebo group during both positions, but was significantly increased in the atropine group during the standing session $(0.55 \pm 0.14)$, compared with during the sitting session $(0.45 \pm 0.14, \mathrm{P}=0.006)$. 

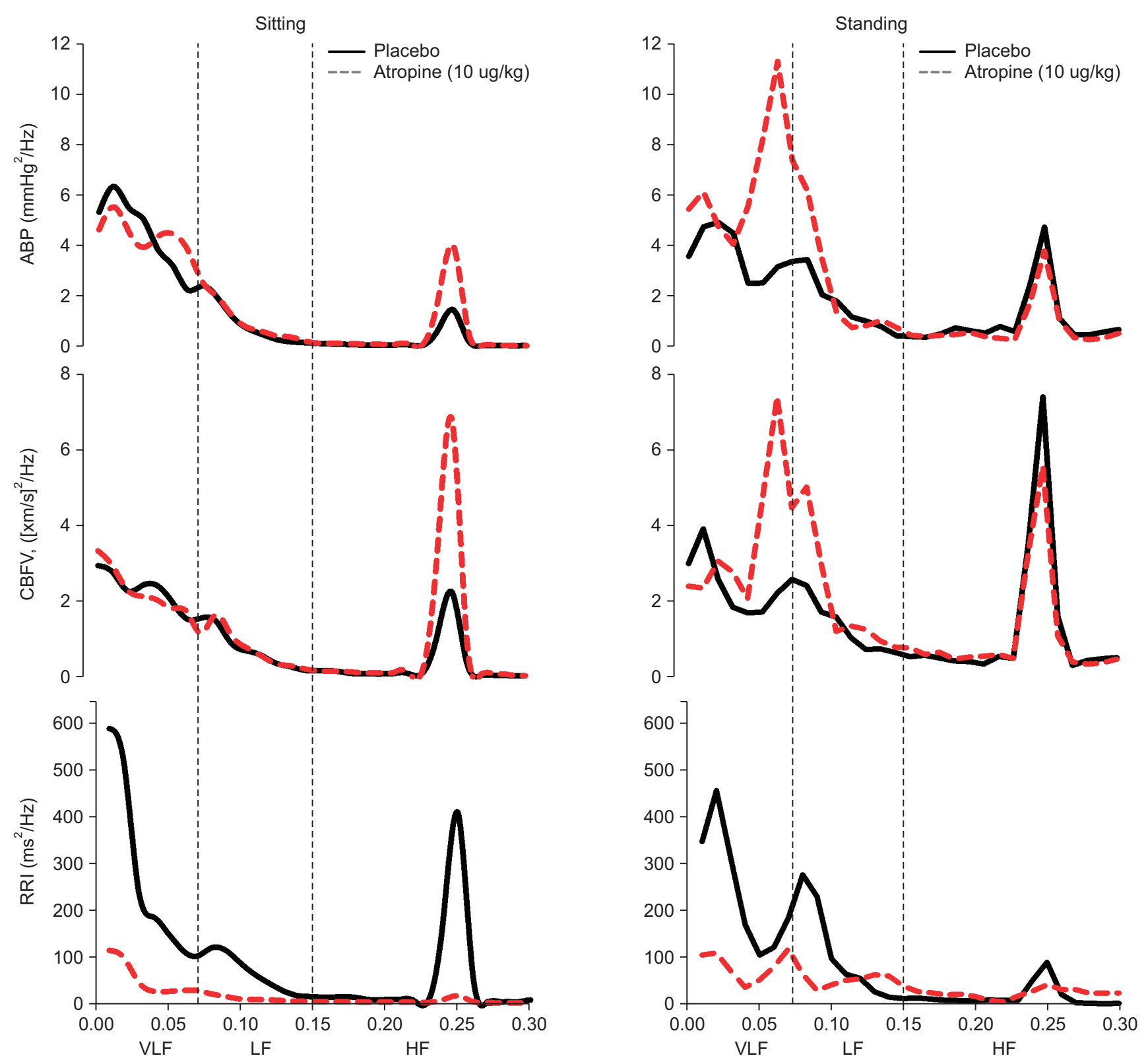

Fig. 4. Group-averaged spectral power density of arterial blood pressure (ABP), cerebral blood flow velocity (CBFV) and R-R interval (RRI) in the very-low-frequency range (VLF, $0.02-0.07 \mathrm{~Hz}$ ), low-frequency range (LF, 0.07-0.15 Hz), and high-frequency range $(\mathrm{HF}, 0.15-0.30 \mathrm{~Hz})$ during both sitting and standing positions. In the atropine group, LFABP and LFCBFV spectral powers were significantly increased in the standing position $(\mathrm{P}<$ 0.05 , respectively) compared with the sitting position. LFABP spectral power in the standing position was higher significantly in the atropine group than the placebo $(\mathrm{P}=0.008)$.

In the standing position, VLF of coherence in the atropine group was increased compared with the placebo group $(0.55 \pm 0.14$ vs. $0.47 \pm 0.16, \mathrm{P}=0.074)$. Gain and phase in the VLF range were not different in the both groups.

\section{Discussion}

We found in this study that vagolytic atropine $(10 \mu \mathrm{g} / \mathrm{kg})$ causes CBFV to fall significantly more as compared with a placebo under sudden postural decreases in ABP. Also, the decreased CBFV during standing was delayed a recovery in the atropine group. Reduction in CVR was significantly lesser in the atropine group than the placebo. Although Gain and Phase in the VLF range were not significantly changed with administration of atropine, transfer function coherence increased during the standing session. Considered together, these findings suggest that the vagolytic effect of atropine attenuates cerebral vasodilation response, and may fall cerebrovascular reactivity during 

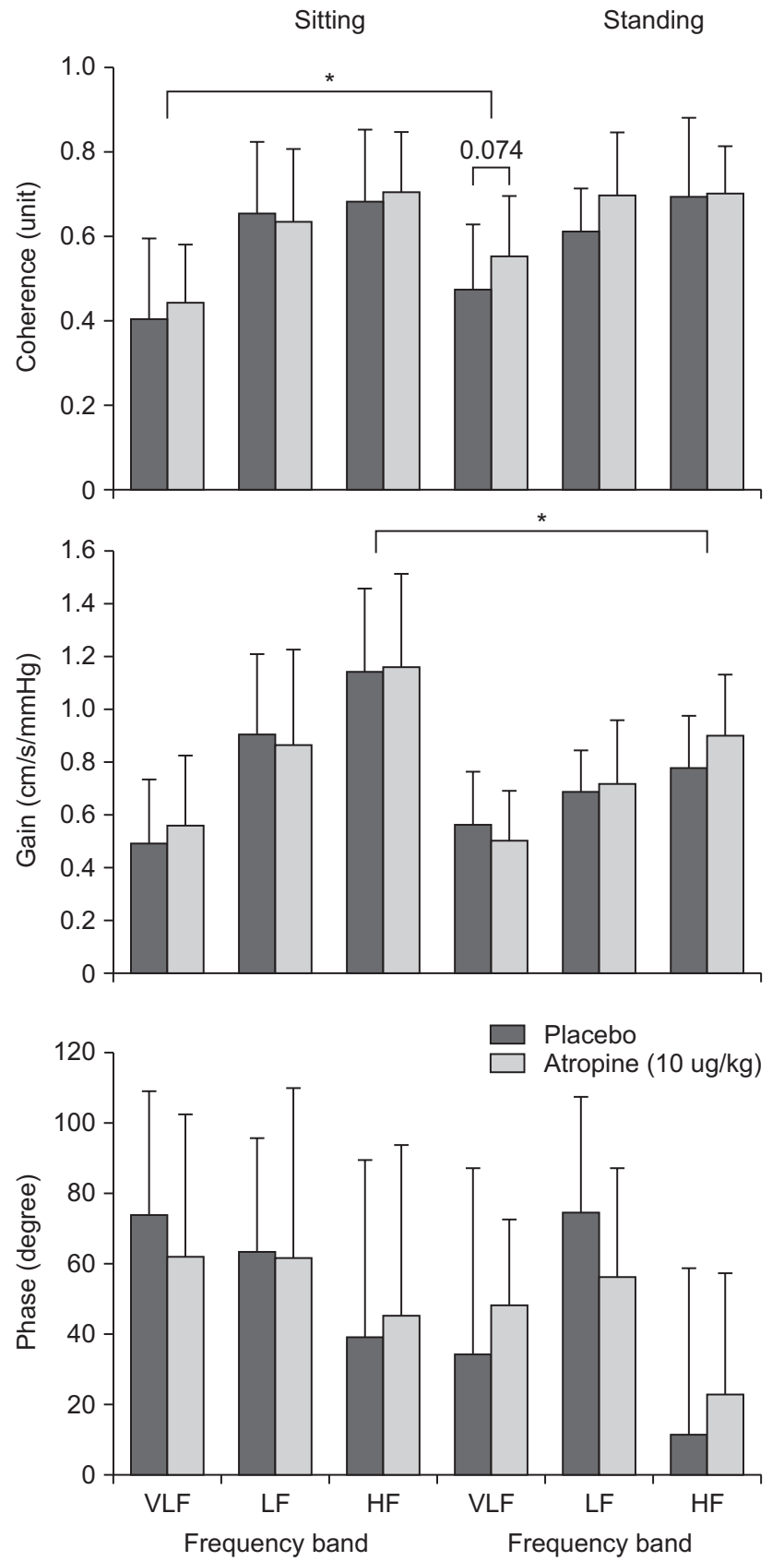

Fig. 5. Transfer function coherence, gain, and phase relating fluctuations in arterial blood pressure and cerebral blood flow velocity in the verylow-frequency range (VLF, $0.02-0.07 \mathrm{~Hz}$ ), low-frequency range (LF, $0.07-0.15 \mathrm{~Hz}$ ), and high-frequency range (HF, 0.15-0.30 Hz) during both sitting and standing positions. Coherence in the VLF range was significantly increased above 0.5 in the atropine group during the standing position. Values are means $\pm \mathrm{SD}$. ${ }^{*} \mathrm{P}<0.05$ vs. Sitting.

orthostatic stress.

Concerning brain muscarinic receptors, heterogenous subtypes of muscarinic receptor have been identified in human brain microvessels [19]. The cholinergic dilation of cerebral vessels is abolished in M5 muscarinic acetylcholine receptor knock- out mice [20], indicating that acetylcholine is an important regulator of local CBF for intraparenchymal brain microvessels. Furthermore, in Alzheimer's disease, perivascular cholinergic nerve terminals of cerebral microvessels are largely lost, and the cortical CBF is diminished [4,21]. Clinically, cholinesterase inhibitors have the effect on cerebrovascular reactivity and neurovascular coupling [5,22], and are commonly used for the treatment in the patients of Alzheimer's disease. In the present study, we infused atropine which can penetrate the blood-brain barrier; therefore, atropine is believed to act on muscarinic receptors within the brain parenchyma, as well as on cerebral vessels at the brain surface. Consequently, reduction of CBFV in the atropine group during the sit-to-stand procedure may be due to changes in cerebrovascular tone from cerebral vessels both at the surface and within the brain.

There are several possible explanations for the reduction in CBFV during orthostatic hypotension after $10 \mu \mathrm{g} / \mathrm{kg}$ atropine application. First, the vagolytic effect of atropine attenuate the vasodilatory response of cerebral artery by acetylcholine $[6,20]$. Second, the parasympathetic block and/or sympathetic activation caused by standing procedure may cause a dynamic shift in the autoregulatory range to the right (higher pressures). This shift may compromise CA during hypotension induced by orthostatic stress, contributing to symptoms of presyncope [23]. In an animal study [24], the lower limit of CA is shifted towards higher blood pressures in parasympathetically denervated rats. Thus, a vagolytic effect of atropine might also cause the shift to the right in the autoregulatory curve relating $\mathrm{ABP}$ to $\mathrm{CBF}$, and might attenuate the autoregulatory response during the standing. Another explanation is that the reduction of $\mathrm{HR}$ variability and cardiac baroreflex caused by vagolytic atropine may affect the changes in CBFV during the change of posture. Previous studies suggests $H R$ variability may contribute to changes in CBFV independent of its effects on ABP variability [25], and that acute cardiac baroreflex dysfunction resulting from cardiac autonomic blockade attenuates dynamic CA [26].

In this study, in addition to studying the effects of orthostatic hypotension, we also used transfer function analysis to evaluate the effect of atropine on dynamic CA during spontaneous fluctuation of ABP. During the sitting session, coherence in the VLF range was not different in both groups. However, during the standing session, VLF of coherence in the atropine group was significantly increased above 0.5 compared with the sitting position and the placebo group. That is, the cerebrovascular response in the atropine group was more passively or linearly to changes in pressure than in the placebo group. Although Gain and Phase in the VLF range were not significantly changed, this increase of coherence with cholinergic blockade may attenuate dynamic CA.

The primary limitation of the current study is the use of tran- 
scranial Doppler ultrasonography for measurement of middle CBF. For velocity changes to be equivalent to flow changes, the diameter of the MCA should remain constant. Numerous studies have shown that MCA diameter in humans remains relatively constant under a variety of hemodynamic conditions [27,28]. Therefore, we assumed that beat-to-beat changes in CBFV in the present study reflected primarily changes in blood flow.

Another limitation is the dosage $(10 \mu \mathrm{g} / \mathrm{kg})$ of atropine used in this study. The dosage of atropine used clinically can be up to as much as $40 \mu \mathrm{g} / \mathrm{kg}$. Although it is known that $10-15 \mu \mathrm{g} / \mathrm{kg}$ atropine is sufficient to block the vagus nerve [10,29], we cannot exclude the possibility that a higher dose of atropine may be needed to completely block the cholinergic nerve system [30]. Finally, the subjects in this study were healthy volunteers. For further evaluation of the effects of atropine on cerebral perfusion, it may be useful to study subjects with compromised hemodynamics or cerebrovascular disease.

In conclusion, the present study shows that cholinergic block by $10 \mu \mathrm{g} / \mathrm{kg}$ atropine attenuated cerebral vasodilation response to acute orthostatic hypotension, causing CBFV to fall significantly more as compared with a placebo. Our findings have implications for further studies of cerebrovascular tone in various clinical conditions associated with vagal impairment.

\section{References}

1. Paulson OB, Strandgaard S, Edvinsson L. Cerebral autoregulation. Cerebrovasc Brain Metab Rev 1990; 2: 161-92.

2. Suzuki N, Hardebo JE, Kåhrström J, Owman C. Selective electrical stimulation of postganglionic cerebrovascular parasympathetic nerve fibers originating from the sphenopalatine ganglion enhances cortical blood flow in the rat. J Cereb Blood Flow Metab 1990; 10: 383-91.

3. Tsukahara T, Usui H, Taniguchi T, Shimohama S, Fujiwara M, Handa H. Characterization of muscarinic cholinergic receptors in human and dog cerebral arteries. Stroke 1986; 17: 300-5.

4. Iadecola C. Neurovascular regulation in the normal brain and in Alzheimer's disease. Nat Rev Neurosci 2004; 5: 347-60.

5. Bar KJ, Boettger MK, Seidler N, Mentzel HJ, Terborg C, Sauer H. Influence of galantamine on vasomotor reactivity in Alzheimer's disease and vascular dementia due to cerebral microangiopathy. Stroke 2007; 38: 3186-92.

6. Seifert T, Fisher JP, Young CN, Hartwich D, Ogoh S, Raven PB, et al. Glycopyrrolate abolishes the exercise-induced increase in cerebral perfusion in humans. Exp Physiol 2010; 95: 1016-25.

7. Hamner JW, Tan CO, Tzeng YC, Taylor JA. Cholinergic control of the cerebral vasculature in humans. J Physiol 2012; 590: 6343-52.

8. Proakis AG, Harris GB. Comparative penetration of glycopyrrolate and atropine across the blood--brain and placental barriers in anesthetized dogs. Anesthesiology 1978; 48: 339-44.

9. Hamel E. Perivascular nerves and the regulation of cerebrovascular tone. J Appl Physiol (1985) 2006; 100: 1059-64.

10. Keyl C, Schneider A, Dambacher M, Bernardi L. Time delay of vagally mediated cardiac baroreflex response varies with autonomic cardiovascular control. J Appl Physiol (1985) 2001; 91: 283-9.

11. Aaslid R, Markwalder TM, Nornes H. Noninvasive transcranial Doppler ultrasound recording of flow velocity in basal cerebral arteries. J Neurosurg 1982; 57: 769-74.

12. Lipsitz LA, Mukai S, Hamner J, Gagnon M, Babikian V. Dynamic regulation of middle cerebral artery blood flow velocity in aging and hypertension. Stroke 2000; 31: 1897-903.

13. Serrador JM, Sorond FA, Vyas M, Gagnon M, Iloputaife ID, Lipsitz LA. Cerebral pressure-flow relations in hypertensive elderly humans: transfer gain in different frequency domains. J Appl Physiol (1985) 2005; 98: 151-9.

14. Zhang R, Zuckerman JH, Giller CA, Levine BD. Transfer function analysis of dynamic cerebral autoregulation in humans. Am J Physiol 1998; 274: H233-41.

15. Zhang R, Zuckerman JH, Iwasaki K, Wilson TE, Crandall CG, Levine BD. Autonomic neural control of dynamic cerebral autoregulation in humans. Circulation 2002; 106: 1814-20.

16. Diehl RR, Linden D, Lucke D, Berlit P. Phase relationship between cerebral blood flow velocity and blood pressure. A clinical test of autoregulation. Stroke 1995; 26: 1801-4.

17. Immink RV, van den Born BJ, van Montfrans GA, Koopmans RP, Karemaker JM, van Lieshout JJ. Impaired cerebral autoregulation in patients with malignant hypertension. Circulation 2004; 110: 2241-5.

18. Lee K, Jackson DN, Cordero DL, Nishiyasu T, Peters JK, Mack GW. Change in spontaneous baroreflex control of pulse interval during heat stress in humans. J Appl Physiol (1985) 2003; 95: 1789-98.

19. Elhusseiny A, Cohen Z, Olivier A, Stanimirovic DB, Hamel E. Functional acetylcholine muscarinic receptor subtypes in human brain microcirculation: identification and cellular localization. J Cereb Blood Flow Metab 1999; 19: 794-802.

20. Yamada M, Lamping KG, Duttaroy A, Zhang W, Cui Y, Bymaster FP, et al. Cholinergic dilation of cerebral blood vessels is abolished in M(5) muscarinic acetylcholine receptor knockout mice. Proc Natl Acad Sci U S A 2001; 98: 14096-101.

21. Tong XK, Hamel E. Regional cholinergic denervation of cortical microvessels and nitric oxide synthase-containing neurons in Alzheimer's disease. Neuroscience 1999; 92: 163-75. 
22. Rosengarten B, Paulsen S, Molnar S, Kaschel R, Gallhofer B, Kaps M. Acetylcholine esterase inhibitor donepezil improves dynamic cerebrovascular regulation in Alzheimer patients. J Neurol 2006; 253: 58-64.

23. Levine BD, Giller CA, Lane LD, Buckey JC, Blomqvist CG. Cerebral versus systemic hemodynamics during graded orthostatic stress in humans. Circulation 1994; 90: 298-306.

24. Morita Y, Hardebo JE, Bouskela E. Influence of cerebrovascular parasympathetic nerves on resting cerebral blood flow, spontaneous vasomotion, autoregulation, hypercapnic vasodilation and sympathetic vasoconstriction. J Auton Nerv Syst 1994; 49 Suppl: S9-14.

25. Cencetti S, Lagi A, Cipriani M, Fattorini L, Bandinelli G, Bernardi L. Autonomic control of the cerebral circulation during normal and impaired peripheral circulatory control. Heart 1999; 82: 365-72.

26. Ogoh S, Tzeng YC, Lucas SJ, Galvin SD, Ainslie PN. Influence of baroreflex-mediated tachycardia on the regulation of dynamic cerebral perfusion during acute hypotension in humans. J Physiol 2010; 588: 365-71.

27. Serrador JM, Picot PA, Rutt BK, Shoemaker JK, Bondar RL. MRI measures of middle cerebral artery diameter in conscious humans during simulated orthostasis. Stroke 2000; 31: 1672-8.

28. Larsen FS, Olsen KS, Hansen BA, Paulson OB, Knudsen GM. Transcranial Doppler is valid for determination of the lower limit of cerebral blood flow autoregulation. Stroke 1994; 25: 1985-8.

29. Montano N, Cogliati C, Porta A, Pagani M, Malliani A, Narkiewicz K, et al. Central vagotonic effects of atropine modulate spectral oscillations of sympathetic nerve activity. Circulation 1998; 98: 1394-9.

30. Bernardi L, Bianchini B, Spadacini G, Leuzzi S, Valle F, Marchesi E, et al. Demonstrable cardiac reinnervation after human heart transplantation by carotid baroreflex modulation of RR interval. Circulation 1995; 92: 2895-903. 